\title{
Sataloff RT, Chowdhury F, Portnoy JE, Hawkshaw MJ, Joglear S: Surgical techniques in otolaryngology-head and neck surgery-laryngeal surgery
}

\author{
Susanne Wiegand
}

Published online: 31 May 2014

(C) Springer-Verlag Berlin Heidelberg 2014

Laryngeal surgery is part of a comprehensive series entitled "Surgical techniques in otolaryngology-head and neck surgery". Contents of the laryngeal surgery volume are organized into five sections. The first section provides a brief introduction to the history of phonosurgery, anatomy and physiology of the larynx, laryngoscopy, aspects of patient selection, preoperative voice evaluation, selection of instrumentation, laryngeal laser surgery and postoperative voice care. Sections $2-5$ offer information of evaluation and treatment of benign structural lesions, premalignant and malignant lesions of the larynx, neurogenic voice disorders and laryngeal trauma. Attention to detail is central to the allure of this book, with comprehensive step-by-step explanations of surgical techniques. The clearly structured book covers the full range of laryngeal procedures and gives readers excellent insight into the complex field of laryngeal surgery with high-performance illustrations supplementing the text throughout the book. Each chapter offers background information and a number of intraoperative pictures guiding the surgeon interested in performing laryngeal surgery. The accompanying DVD includes 13 video clips and slide shows including arytenoid mobilization, cyst excision, scar release and different laser therapies. The book is intended as a practical resource for physicians in practice and those in training. In summary, the book together with the video demonstrations can be considered as an excellent manual of which also experienced otolaryngologist benefits largely. Without any doubt it is a gain for the book market in the field of laryngeal surgery.
S. Wiegand $(\bowtie)$

Department of Otolaryngology, Head \& Neck Surgery, University

of Marburg, Marburg, Germany

e-mail: swiegand@med.uni-marburg.de 